\title{
NEUROIMMUNOLOGY
}

\section{IL-17A mediates a path to autism}

Severe viral infection during pregnancy has been linked to increased risk of autism spectrum disorder (ASD) in offspring. Now, a study in mice shows that ASD-like behaviour in offspring in response to maternal immune activation depends on maternal $\mathrm{CD} 4^{+} \mathrm{T}$ cells expressing retinoic acid receptor-related orphan receptor- $\gamma \mathrm{t}(\mathrm{ROR} \gamma \mathrm{t})$ and interleukin-17A (IL-17A); blocking this pathway restored normal behaviour and brain structure.

T helper $17\left(\mathrm{~T}_{\mathrm{H}} 17\right)$ cells have been implicated in ASD as IL-17A can be detected in the blood in a subset of children with ASD, and IL17A is one of the many genes linked to susceptibility to ASD; however, whether $\mathrm{T}_{\mathrm{H}} 17$ cells promote ASD has been unclear. To study the underlying mechanisms causing ASD-like behaviour in mice, Choi et al. injected pregnant mice with polyinosinicpolycytidylic acid (poly(I:C)) to c4

the maternal

IL-17A

pathway

mediates

irregular brain

structure mimic viral infection and induce maternal immune activation. At embryonic day 14.5 (E14.5), mothers had increased serum levels of IL-17A and elevated mRNA levels of $I l 17 a$ in placental- and decidua-associated mononuclear cells. Furthermore, offspring from these mice showed increased mRNA expression of the IL-17A receptor in the brain.

Next, the authors investigated whether activation of the IL-17A pathway in pregnant mice affects fetal brain development and thereby contributes to ASD-like behaviour in offspring. Analysis of cortical layer-specific markers at E14.5 and E18.5 revealed irregularities in the normally well-defined layers of neurons in the brain cortex in offspring from mice injected with poly(I:C). This was not observed in offspring from control mice or offspring from poly(I:C)-injected mothers pretreated with IL-17A-blocking antibodies. Behavioural studies showed that IL-17A-induced signalling was associated with deficits in social interaction and with repetitive behaviour and abnormal communication in offspring of poly(I:C)injected pregnant mice. These behavioural defects were corrected in offspring from mothers pretreated with IL-17A-blocking antibodies. Thus, the maternal IL-17A pathway mediates irregular brain structure and behavioural abnormalities in offspring from mice exposed to maternal immune activation.

So, could maternal expression of ROR $\gamma \mathrm{t}$ - which is an important regulator of the IL-17A pathway have a role in the behavioural phenotypes of offspring from mothers exposed to immune activation? Female mice with a T cell-specific ROR $\gamma t$ deficiency were mated with wild-type male mice, so that offspring had at least one functional copy of ROR $\gamma t$ and functional $\mathrm{T}_{\mathrm{H}} 17$ cells. Pregnant ROR $\gamma$ t-deficient mice failed to produce IL-17A after poly(I:C) injection, and offspring from these mice showed normal cortex structure and normal behaviour. Hence, maternal ROR $\gamma \mathrm{t}$-expressing $\mathrm{CD}^{+} \mathrm{T}$ cells, such as $\mathrm{T}_{\mathrm{H}} 17$ cells, are required for the ASD-like behaviours seen in mouse offspring after maternal immune activation.

Finally, the authors found that treating pregnant mice with IL-17Ablocking antibodies after induction of maternal immune activation could correct some of the ASD-like behaviours, but pretreatment with this antibody had a greater effect and therefore might have more therapeutic potential. The future will tell whether these findings can be translated to humans.

Elisabeth Kugelberg

ORIGINAL ARTICLE Choi, G. B. et al.

The maternal interleukin-17a pathway in mice promotes autism-like phenotypes in offspring. Science 351, 933-939 (2016) 